\title{
Network-Level Dysconnectivity in Drug-Naïve First-Episode Psychosis: Dissociating Transdiagnostic and Diagnosis-Specific Alterations
}

\author{
Qiyong Gong ${ }^{1,2,3}$, Xinyu Hu', William Pettersson-Yeo ${ }^{4}, X_{i n} \mathbf{X u}^{3}$, Su Lui', Nicolas Crossley ${ }^{4,5}$, Min Wu', \\ Hongyan Zhu',6 and Andrea Mechelli ${ }^{4}$ \\ 'Huaxi MR Research Center (HMRRC), Departments of Radiology, West China Hospital of Sichuan University, Chengdu, China; ${ }^{2}$ Department of \\ Psychology, School of Public Administration, Sichuan University, Chengdu, China; ${ }^{3}$ Department of Psychiatry, West China Hospital of Sichuan \\ University, Chengdu, China; ${ }^{4}$ Department of Psychosis Studies, Institute of Psychiatry, Psychology and Neuroscience, King's College London, London, \\ UK; ${ }^{5}$ Department of Psychiatry, School of Medicine, Pontifical Catholic University of Chile, Santiago, Chile; 'aboratory of Stem Cell Biology, State \\ Key Laboratory of Biotherapy, West China Hospital of Sichuan University, Chengdu, China
}

\begin{abstract}
The neuroimaging literature provides compelling evidence for functional dysconnectivity in people with psychosis. However, it is likely that at least some of the observed alterations represent secondary effects of illness chronicity and/or antipsychotic medication. In addition, the extent to which these alterations are specific to psychosis or represent a transdiagnostic feature of psychiatric illness remains unclear. The aim of this study was therefore to examine the diagnostic specificity of functional dysconnectivity in drug-naïve first-episode psychosis (FEP). We used resting-state functional magnetic resonance imaging and functional connectivity analysis to estimate network-level connectivity in 50 patients with FEP, 50 patients with major depressive disorder (MDD), 50 patients with post-traumatic stress disorder (PTSD), and 122 healthy controls (HCs). The FEP, MDD, and PTSD groups showed reductions in intranetwork connectivity of the default mode network relative to the HC group ( $p<0.05$ corrected); therefore, intranetwork alterations were expressed across the three diagnostic groups. In addition, the FEP group showed heightened internetwork connectivity between the default mode network, particularly the anterior cingulate cortex, and the central executive network relative to the MDD, PTSD, and HC groups $(p<0.05$ corrected); therefore, internetwork alterations were specific to the FEP. These findings suggest that network-level alterations are present in individuals with a first episode of psychosis who have not been exposed to antipsychotic medication. In addition, they suggest a dissociation between aberrant internetwork connectivity as a distinctive feature of psychosis and aberrant intranetwork connectivity as a transdiagnostic feature of psychiatric illness.

Neuropsychopharmacology (2017) 42, 933-940; doi:I 0.I 038/npp.20 I6.247; published online 16 November 2016
\end{abstract}

\section{INTRODUCTION}

Although it is recognized that psychosis is associated with abnormalities in specific regions of the brain, it has not been possible to explain its complex clinical features on the basis of localized deficits. This has led to the hypothesis that the core neural deficit of this disorder lies in abnormal functional integration within large-scale neuronal networks (Friston, 1998; Pettersson-Yeo et al, 2011; Schmidt et al, 2014). Over the past two decades, the development of neuroimaging techniques, such as resting-state functional connectivity magnetic resonance imaging (rs-fcMRI), has provided an opportunity to investigate this hypothesis in

*Correspondence: Professor H Zhu, Laboratory of Stem Cell Biology, West China Hospital of Sichuan University, 37 Guoxue Lane, Wuhou District, Chengdu, Sichuan Province, 6I 004I, China, Tel/Fax: +86 (0) 28 85423503, E-mail: hyzhu_hmrr@@|26.com

Received 25 April 2016; revised 6 October 2016; accepted 17 October 2016; accepted article preview online 26 October 2016 detail (Fornito and Bullmore, 2015; Pettersson-Yeo et al, 2011; Rubinov and Bullmore, 2013b; van den Heuvel and Fornito, 2014). This has revealed aberrant connectivity in several networks including, amongst others, the default mode network (DMN), central executive network (CEN), and salience network (SN) of patients with psychosis (Menon, 2011; Woodward and Cascio, 2015).

The DMN is involved in the monitoring of internal processes including autobiographical and self-monitoring, and is typically deactivated during stimulus-driven, externally oriented cognitive tasks; it includes the anterior and posterior cingulate cortex, the medial prefrontal cortex, the medial temporal lobe, and the angular gyrus (Littow et al, 2015; Menon, 2011). DMN dysfunction is commonly observed in psychosis, and is thought to underlie the deficits in episodic memory and self-monitoring typically observed in this disorder (Littow et al, 2015; Menon, 2011).

The CEN is responsible for high-level cognitive functions including planning, decision-making, working memory, and attentional control in the context of goal-directed behavior; it 
includes the dorsolateral prefrontal cortex and the posterior parietal cortex (Littow et al, 2015; Menon, 2011). As psychosis is characterized by some degree of cognitive dysfunction, it is not surprising that this network is disrupted in this condition (Littow et al, 2015; Menon, 2011).

The SN is involved in detecting, orienting to, and integrating salient external stimuli and internal events such as interoceptive, autonomic, and emotional information; it comprises the dorsal anterior cingulate cortex, the frontoinsular cortex, the amygdale, and the substantia nigra/ventral tegmental area. Impaired processing of salient external stimuli and internal events is thought to affect both cognition and self-monitoring in psychosis (Menon, 2011; Menon and Uddin, 2010).

Taken collectively, the above findings suggest that DMN, $\mathrm{CEN}$, and SN dysfunction is a prominent feature of psychosis (Nekovarova et al, 2014). The significance and translational applicability of these findings, however, is unclear because of two critical limitations in the existing literature. First, apart from few notable exceptions ( $\mathrm{Du}$ et al, 2015), the vast majority of studies compared a group of patients against a control group of healthy individuals; therefore, it was not possible to establish whether any observed differences were specific to psychosis or represented a general feature of psychiatric illness. From a clinical translation perspective, the real challenge is not to distinguish between patients and healthy individuals, but to develop differential biomarkers that could be used to distinguish between alternative diagnoses (Kapur et al, 2012). Second, the vast majority of previous studies examined patients with established psychosis who had been on antipsychotic medication for several weeks, months, or even years. This means that some of the network-level alterations reported in these studies might reflect secondary effects of illness chronicity and/or antipsychotic medication (Sarpal et al, 2015).

Here we attempted to address the limitations of the existing literature by investigating network-level functional connectivity in 50 drug-naive patients with a diagnosis of first-episode psychosis (FEP), 50 drug-naïve patients with a diagnosis of major depressive disorder (MDD), 50 drugnaive patients with a diagnosis of post-traumatic stress disorder (PTSD), and 122 healthy individuals free from psychiatric diagnosis (healthy controls (HCs)). All participants were examined with rs-fcMRI using the same scanner and acquisition sequence, allowing the direct comparison between the different diagnostic groups. We hypothesized that (i) patients with FEP would express lower withinnetwork connectivity, particularly within the DMN given the use of a resting-state paradigm (Pettersson-Yeo et al, 2011), than HCs; (ii) patients with FEP would express heightened internetwork connectivity, particularly between the DMN and the CEN consistent with previous investigations that used the same resting-state paradigm (Manoliu et al, 2014; Williamson, 2007) relative to HCs; (iii) some of these changes would also be expressed in the MDD and PTSD groups, whereas others would be specific to patients with FEP.

\section{MATERIALS AND METHODS}

\section{Participants}

Subsets of the data used here have been used in previous studies (Jin et al, 2014; Lui et al, 2011; Ren et al, 2013). The present investigation was approved by the local research ethics committee and written informed consent was obtained from all participants. Exclusion criteria applicable to all participants included (i) history of drug or alcohol abuse, (ii) pregnancy, and (iii) any physical illness such as hepatitis, cardiovascular disease, or neurological disorder, as assessed by interview and review of medical records. While cannabis use was not an exclusion criteria, none of the participants reported being previous or current users. More information on each group is provided below.

\section{First-Episode Psychosis}

Fifty patients with FEP were included in the analysis here (see Table 1). Diagnosis of FEP and duration of illness were determined by the consensus of two experienced clinical psychiatrists using the Structured Interview for the DSM-IV Axis I Disorder, Patient Edition (SCID) (First et al, 1997). Psychopathology was measured on the day of scanning using the Positive and Negative Syndrome Scale (PANSS) (Kay et al, 1987). At the time of scanning, all patients were medication-naïve.

\section{Major Depressive Disorder}

Fifty participants with MDD were included in the analysis here (see Table 1). These participants were part of a larger cohort study of major depression in the Chinese population of Han nationality. Diagnosis of MDD was made with the

Table I Demographic and Clinical Characteristics of Participants

\begin{tabular}{|c|c|c|c|c|c|}
\hline Subject group & FEP $(n=50)$ & MDD $(n=50)$ & PTSD $(n=50)$ & $\mathrm{HC}(n=122)$ & Group comparison \\
\hline Age $( \pm$ SEM $)$ & $32.72(1.03)$ & $38.89(1.64)$ & $43.10(1.53)$ & $29.61(1.30)$ & $F=17.30$, d.f. $=3, p<0.05$ \\
\hline Gender & I6M:34F & 16M:34F & I6M:34F & $58 \mathrm{M}: 64 \mathrm{~F}$ & $X^{2}=6.83$, d.f. $=3, p=0.078$ \\
\hline Years of education $( \pm$ SEM) & I $3.28(0.58)$ & I2.83 (0.60) & $6.90(0.49)$ & $12.33(0.29)$ & $\mathrm{F}=36.18, \mathrm{~d} . \mathrm{f} .=3, p<0.05$ \\
\hline Symptom scores ( \pm SEM) & $\begin{array}{c}\text { PANSS positive: } 24.84(0.97) \\
\text { PANSS negative: } 16.84(1.22) \\
\text { PANSS general: } 46.62(1.32) \\
\text { PANSS total: } 88.30(2.77)\end{array}$ & HAM-D: $23.22(0.67)$ & PCL: 5 I.20 (I.48) & & \\
\hline
\end{tabular}

Abbreviations: d.f., degrees of freedom; F, females; FEP, first-episode psychosis; HAM-D, Hamilton Depression Rating Scale; HC, healthy controls; M, males; MDD, major depressive disorder; n, number of subjects; NS, not significant; PANSS, Positive and Negative Syndrome Scale; PCL, PTSD Checklist; PTSD, post-traumatic stress disorder. 
SCID (First et al, 1997). Severity of depression was quantified using the 17-item Hamilton Depression Rating Scale (HAMD) (Williams, 1988); all participants had a total HAM-D score $\geqslant 18$ on the day. At the time of scanning, all patients were medication-naïve.

\section{Post-Traumatic Stress Disorder}

Fifty participants with PTSD were included in the analysis here (Jin et al, 2014) (see Table 1). These participants were survivors of the 2008 Sichuan earthquake who had all physically experienced the earthquake, personally witnessed death, serious injury, or the collapse of buildings and not suffered any physical injury. All participants were assessed using the PTSD Checklist (PCL) (Weathers et al, 1994) and met the threshold criteria (PCL score $\geqslant 35$ ) for diagnosis of PTSD on the day of scanning. At the time of scanning, all patients were medication-naïve.

\section{Healthy Controls}

One hundred and twenty-two HC subjects were included in the analysis here (see Table 1). All were recruited by poster advertisement, and screened using the non-patient edition of the SCID to confirm the lifetime absence of psychiatric illnesses. In addition, these subjects were interviewed to exclude individuals with a known history of psychiatric illness in first-degree relatives.

\section{Data Acquisition}

All neuroimaging was conducted using a 3-T MRI scanner (EXCITE; General Electric, Milwaukee, WI) with an 8channel phased-array head coil. Images were acquired using a gradient-echo echo-planar imaging sequence with a repetition time $(\mathrm{TR})=2000 \mathrm{~ms}$, an echo time $(\mathrm{TE})=30 \mathrm{~ms}$, flip angle $=90^{\circ}$, slice thickness $=5 \mathrm{~mm}$ (no slice gap), $64 \times 64$ matrix, and field of view $=24 \mathrm{~cm}^{2}$, resulting in a voxel size of $3.75 \times 3.75 \times 5 \mathrm{~mm}^{3}$. Scanning lasted for $410 \mathrm{~s}$, with each brain volume comprising 30 axial slices and each functional run 205 image volumes. Participants were fitted with soft earplugs, positioned carefully in the coil with comfortable support, and instructed to relax with their eyes closed without falling asleep; after the experiment, each participant confirmed not having fallen asleep during scanning. The four groups were recruited and scanned over the same period of time (June 2008-May 2012).

\section{Data Analysis}

Preprocessing. Functional images were preprocessed using the SPM8 software (http://www.fil.ion.ucl.ac.uk/spm) running under Matlab 7.1 (Math Works, Natick, MA). Before preprocessing, the first five scans were discarded to remove the impact of magnetization stabilization. For each participant, images were realigned to the first of the run and resliced with sinc interpolation. The resulting images were then normalized to a standard ICBM EPI template using nonlinear-basis functions and spatially smoothed with a 8-mm full-width at half-maximum isotropic Gaussian kernel; this compensated for residual variability in functional anatomy after spatial normalization, and allowed the use of
Gaussian random field theory in the statistical analysis of the data. To test for differences in head movement, we estimated the frame-wise displacement of each subject-a measure of head movement from one volume to the next calculated as the sum of the absolute values of the realignment estimates at every timepoint (Power et al, 2012); an ANOVA did not reveal significant differences across the four groups (d.f. $=3$, $\mathrm{F}=1.903, p=0.129)$.

Time-series construction. Using the preprocessed images, the $\mathrm{T}^{\star}$-informed BOLD signal was extracted from all voxels, and the time-series of the responses were constructed. For each subject, three directional movement parameters were regressed out, in addition to their first derivatives, and cerebrospinal fluid-related signal changes inferred from a mask including only voxels within the ventricles. Finally, we band-passed the time-series between 0.01 and $0.1 \mathrm{~Hz}$.

Network masks. In accordance with our hypotheses, separate analyses were conducted for each network of interest. In each case, a specific mask was used to limit the analysis to those regions included within each network: the DMN, CEN, and salience network (SN), respectively (see Supplementary Figure S1). The masks used here were obtained from a previous study investigating large-scale networks in psychopathology (Menon and Uddin, 2010).

Within-network connectivity. The time-series from each voxel was correlated with the time-series from all other voxels within the same network; this was carried out for each of our networks of interest (ie DMN, CEN, SN). The subsequent correlation matrices were then $\mathrm{z}$-transformed, and the average correlation of each voxel with every other voxel in the mask was calculated. Therefore, for each voxel we obtained a value representing its average correlation with every other voxel in the mask. We then applied a mass-univariate approach looking for voxel-wise differences in the level of coordinated participation within masks between groups, with age and gender included as covariates of no interest. Alterations common to the three diagnostic groups were identified by comparing FEP, MDD, and PTSD patients against healthy controls (eg FEP, MDD, and PTSD $v s \mathrm{HC}$ ) and then using the inclusive masking option (at $p<0.05$ uncorrected) in the SPM8 software to identify those regions that survived the diagnosis-specific comparisons (ie FEP vs HC; MDD vs HC; and PTSD vs HC). This enabled us to detect alterations in the FEP group that reflect a transdiagnostic feature of psychiatric illness. Alterations specific to the FEP group were identified by comparing the FEP patients against the other three groups (eg FEP $v s$ HC, MDD and PTSD) and then using the inclusive masking option (at $p<0.05$ uncorrected) in the SPM8 software to identify those regions that survived the individual comparisons (ie FEP vs HC; FEP vs MDD; FEP vs PTSD). This enabled us to detect alterations that are a specific feature of the FEP group. For each network of interest, statistical inferences were made at $p<0.05$ after family-wise error (FWE) correction and a further correction to account for the number of networks investigated $(n=3)$. All reported regions were localized using the Harvard Cortical and 


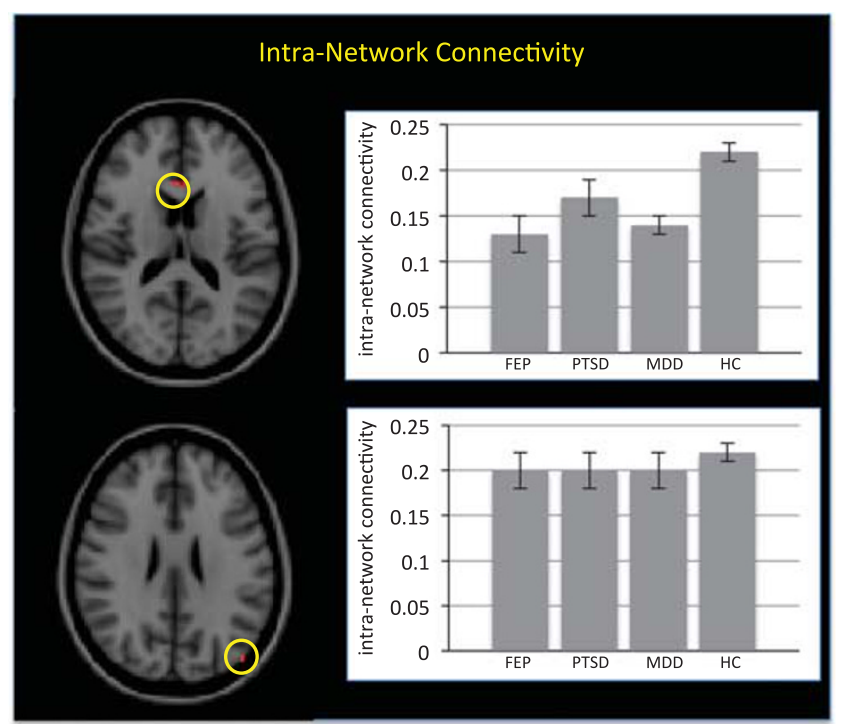

Figure I Transdiagnostic alterations in intranetwork connectivity. Left panels: The two regions showing reduced intranetwork connectivity in the first-episode psychosis (FEP), major depressive disorder (MDD), and posttraumatic stress disorder (PTSD) groups relative to healthy controls (HCs) $(p<0.05$ after family-wise error (FWE) correction). Right panels: Mean intranetwork connectivity for the FEP, MDD, PTSD and HC groups (standard error within parentheses).

Subcortical Atlas toolbox in FMRIB Software Library View (http://fsl.fmrib.ox.ac.uk/fsl/fslwiki/).

Internetwork connectivity. The time-series of each voxel within a network (eg the DMN) was individually correlated with the average time-series of all voxels within another network (eg the SN); this was carried out for each pair of networks (ie DMN-SN, SN-DMN, DMN-CEN, CEN-DMN, SN-CEN and CEN-SN). For each pair, we then applied a mass-univariate approach to test for group differences in functional connectivity between each voxel within the first network (eg the SN) and the second network as a whole (eg the DMN), with age and gender included as covariates of no interest. Alterations common to the three diagnostic groups were identified by comparing FEP, MDD and PTSD patients against HCs (eg FEP, MDD and PTSD vs HC) and then using the inclusive masking option (at $p<0.05$ uncorrected) in the SPM8 software to identify those regions that survived the diagnosis-specific comparisons (ie FEP $v s$ HC; MDD vs $\mathrm{HC}$; and PTSD vs HC). This enabled us to detect alterations in the FEP group that reflect a transdiagnostic feature of psychiatric illness. Alterations specific to the FEP group were identified by comparing the FEP patients against the other three groups (eg FEP vs HC, MDD and PTSD) and then using the inclusive masking option (at $p<0.05$ uncorrected) in the SPM8 software to identify those regions that survived the individual comparisons (ie FEP $v s$ HC; FEP $v s$ MDD and FEP $v s$ PTSD). This enabled us to detect alterations that are a specific feature of the FEP group. For each network of interest, statistical inferences were made at $p<0.05$ after FWE correction and a further correction to account for the number of pairs investigated $(n=6)$. Again, all reported regions were localized using the Harvard Cortical and
Subcortical Atlas toolbox in the FMRIB Software Library View (http://fsl.fmrib.ox.ac.uk/fsl/fslwiki/).

\section{RESULTS}

\section{Demographics}

Demographic information including age, gender, and years of education is reported in Table 1. With respect to age, a one-way ANOVA showed a significant difference between groups $(\mathrm{F}=17.30$, d.f. $=3, p<0.05)$. Post hoc analyses with Bonferroni correction showed that, on average, subjects in the FEP group were significantly younger compared with those in the PTSD group (mean difference $=10.38$ years, $p<0.001$ ). In addition, CTRL subjects were significantly younger compared with those in the PTSD group (mean difference $=13.49$ years, $p<0.001$ ) and the MDD group (mean difference $=9.27$ years, $p<0.001$ ). With respect to gender, a $\chi^{2}$ test showed that there was no significant difference between groups $\left(\chi^{2}=6.83\right.$, d.f. $\left.=3, p=0.078\right)$. With respect to years of education, one-way ANOVA showed a significant difference between groups $(\mathrm{F}=36.18$, d.f. $=3, p<0.05)$. Post hoc tests with Bonferroni correction showed that, on average, subjects in the PTSD group had significantly less years of education on average than those in the FEP group (mean difference $=6.38$ years, $p<0.001$ ), the CTRL group (mean difference $=5.44$ years, $p<0.001$ ), or the MDD group (mean difference $=5.93$ years, $p<0.001$ ). In contrast, there were no significant differences in years of education between the FEP, CTRL, and MDD groups.

\section{Within-Network Connectivity}

The three patient groups showed reduced within-network connectivity relative to the HC group $(p<0.05$ after FWE correction); close inspection of the results revealed that this effect was localized in the ventral-medial prefrontal cortex and the left posterior-inferior parietal cortex within the DMN (see Figure 1 and Table 2). However, it should be noted that the effect in the left posterior-inferior parietal cortex comprised of a single voxel. In contrast, there were no statistically significant differences that were specific to the FEP group. This pattern of results indicates that reduced within-network connectivity is common to the three diagnostic categories rather than a specific feature of the FEP group.

Within-network connectivity in the ventral-medial prefrontal cortex and the left posterior-inferior parietal cortex was not associated with PANSS Positive Score, PANSS Negative Score, PANSS General Score, and PANSS Total Score in the FEP group $(p>0.05)$. In addition, withinnetwork connectivity in the ventral-medial prefrontal cortex and the left posterior-inferior parietal cortex was not correlated ( $p>0.05$ uncorrected) with gray matter volume in the same regions-which was estimated after preprocessing the structural images of all participants using the DARTEL pipeline (Ashburner, 2007); therefore, we found no evidence that our results could be explained by partial volume effects (Pettersson-Yeo et al, 2015). 
Table 2 Transdiagnostic Differences in Intranetwork Connectivity in the FEP, MDD, and PTSD Groups Relative to the HC Group ${ }^{\text {a }}$

\begin{tabular}{|c|c|c|c|c|c|c|c|c|}
\hline Region & $x, y, z$ & FEP & PTSD & MDD & $\mathrm{HC}$ & $K_{\mathrm{c}}$ & z-score & $p$-value \\
\hline Ventral medial prefrontal cortex & $3,23,16$ & $0.13(0.02)$ & $0.17(0.02)$ & $0.14(0.01)$ & $0.22(0.01)$ & 10 & 4.21 & 0.015 \\
\hline Left posterior inferior parietal cortex & $-45,-82,25$ & $0.20(0.02)$ & $0.20(0.02)$ & $0.20(0.02)$ & $0.22(0.01)$ & । & 4.16 & 0.018 \\
\hline
\end{tabular}

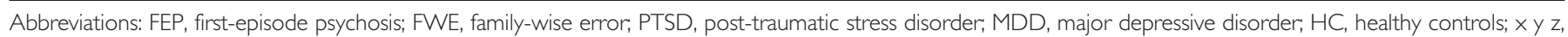
Montreal Neurological Institute coordinates; $K_{C}$, cluster size.

All p-values are reported after FWE correction for multiple comparisons.

${ }^{a}$ In contrast, there were no disease-specific differences in intranetwork connectivity in the FEP group relative to MDD, PTSD and HC groups.

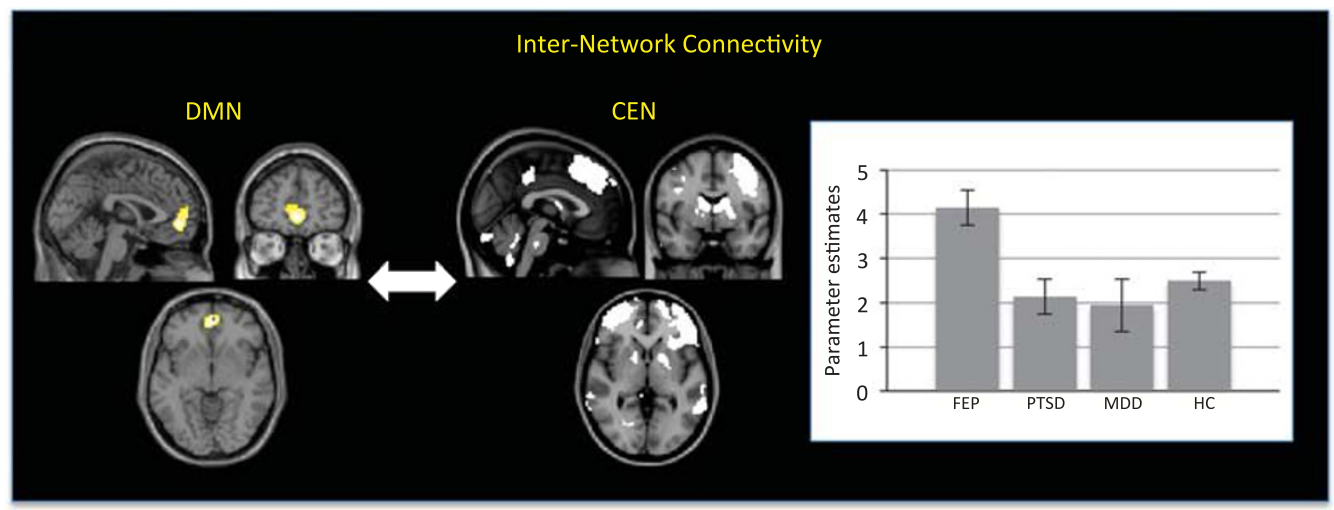

Figure 2 Diagnosis-specific alterations in internetwork connectivity. Left panel: Anterior cingulate region of the default mode network $(D M N)(x=0$, $z=50, z=-5)$, which showed heightened internetwork correlation with the central executive network (CEN) in the first-episode psychosis (FEP) group relative to the major depressive disorder (MDD), post-traumatic stress disorder (PTSD), and healthy control (HC) groups ( $P<0.05$ after family-wise error (few) correction). Right panel: Parameter estimates for the internetwork correlation between the anterior cingulate cortex and the CEN in the FEP, MDD, PTSD, and HC groups (standard error within parentheses).

\section{Internetwork Connectivity}

The FEP group showed significantly greater internetwork correlation between the DMN, particularly the anterior cingulate cortex, and the CEN relative to the other three groups $(\mathrm{x}=0, \mathrm{y}=50, \mathrm{z}=-5$; $z$-score: $5.41, p<0.001$ after FWE correction; cluster size: 162; Figure 2). Individual comparisons revealed that this effect was driven by the FEP group having significantly greater internetwork correlation between the anterior cingulate cortex and the CEN compared with the CTRL group ( $z$-score: $3.91, p=0.012$ after FWE correction), the PTSD group ( $z$-score: 5.17, $p<0.001$ after FWE correction), and the MDD group ( $z$-score: 4.49, $p=0.005$ after FWE correction). In contrast, there was no significant difference between the CTRL, PTSD, and MDD groups $(p>0.05)$. This pattern of results indicates that greater correlation between the anterior cingulate cortex within the DMN and the CEN is specific to the FEP group rather than a common feature of all three diagnostic categories. There were no statistically significant differences in terms of internetwork correlation between the central executive and SNs or between the default mode and SNs.

The internetwork correlation between the anterior cingulate cortex and the CEN was not associated with PANSS Positive Score, PANSS Negative Score, PANSS General Score, and PANSS Total Score in the FEP group $(p>0.05)$. In addition, the internetwork correlation between the anterior cingulate cortex and the CEN was not correlated
( $p>0.05$ FWE-corrected) with regional gray matter volume within these two networks; again, this suggests that our results are unlikely to be explained by partial volume effects (Pettersson-Yeo et al, 2015).

\section{Global Signal}

The average functional connectivity between all gray matter voxels did not differ among the four groups (d.f. $=3$, $\mathrm{F}=2.079, p=0.103)$; therefore, we found no evidence that the above differences in intra- and internetwork connectivity can be explained by differences in global signal.

\section{DISCUSSION}

It has been proposed that the core neural deficit of psychosis lies in aberrant functional integration within large-scale neuronal networks (Friston, 1998; Pettersson-Yeo et al, 2011; Schmidt et al, 2014). Network-level alterations, however, have been observed in several psychiatric disorders, including, for example, psychosis, MDD, and PTSD (Gong and He, 2015; Menon, 2011; Rubinov and Bullmore, 2013a; Woodward and Cascio, 2015; Zhang et al, 2016). This suggests that at least some of the network-level alterations observed in people with psychosis may represent a transdiagnostic feature of psychiatric illness rather than a distinctive feature of the disorder. A further complication is 
that the vast majority of studies examined patients with established psychosis who had been on antipsychotic medication; this raises the possibility that some of the observed changes may have been due to the effects of illness chronicity and/or treatment (Sarpal et al, 2015). To address these limitations, we examined functional connectivity in drug-naïve patients with a first episode of the illness in comparison with HCs, patients with MDD, and patients with PTSD.

First, we characterized functional integration within networks by measuring the average correlation between a voxel and every other voxel in the same functional network. Here the three patient groups showed reduced within-network connectivity relative to the HC group in the ventral-medial prefrontal cortex and the left posterior-inferior parietal cortex within the DMN. Therefore, aberrant functional integration within this network appears to be a transdiagnostic feature of psychiatric illness rather than a distinctive feature of psychosis. The observation of abnormal within connectivity in the DMN but not in the SN or the CEN might be explained by the use of the resting-state paradigm, which involves internal monitoring processes subserved by the DMN. Consistent with this explanation, neuroimaging studies of psychosis that reported dysfunction in the SN or the CEN typically involved an active task and/or the presentation of external stimuli (Menon, 2011). There are, however, at least two studies that reported dysfunction in the $\mathrm{SN}$ or the CEN of people with psychosis during rest (Pelletier-Baldelli et al, 2015; Sridharan et al, 2008; Wang et al, 2016), suggesting that further research is required to elucidate the impact of different experimental paradigms (ie resting state $v s$ active task) on the results.

If poor functional integration of the DMN is a transdiagnostic feature of psychosis, MDD and PTSD, what is the common phenotype of this neural deficit? Psychosis, MDD, and PTSD have in common a significant degree of cognitive dysfunction, which can take different forms including through disorder, catastrophic belief, misinterpretation of external or internal stimuli, and generalization (Power et al, 2012). Consistent with this observation, cognitive dysfunction is a major risk factor for all forms of psychopathology during the complex transition from adolescence to adulthood, and a negative prognostic factor in those individuals who become ill (Pettersson-Yeo et al, 2015). Therefore, we speculate that the functional dysconnectivity of the DMN may underlie the significant degree of cognitive dysfunction that characterizes psychosis, MDD and PTSD. Unfortunately, this possibility could be tested using the present data, as cognitive performance was not assessed.

A characterization of functional integration in terms of intranetwork connectivity can only provide partial information, given the reliance of high-level affective and cognitive processes on the dynamic interaction of multiple networks (Berman et al, 2016; Kelly et al, 2008). Therefore, we also characterized internetwork integration by correlating the average time-series of different networks. This revealed significantly heightened internetwork correlation between the DMN, particularly the anterior cingulate cortex, and the CEN in patients with FEP. The anterior cingulate cortex has long been thought to have a key role in the neuropathology of psychosis; in particular, human and animal studies provide converging evidence for reductions in neuronal, synaptic, and dendritic density, as well as excess of afferent input (Adams and David, 2007; Fornito et al, 2009); interestingly, some of these changes have been shown to normalize upon administration of antipsychotic medication (Braus et al, 2002). The observation of heightened internetwork correlation between the anterior cingulate cortex and the CEN is consistent with the results of previous studies that reported aberrant integration between the DMN and CEN in schizophrenia (Manoliu et al, 2014; Williamson, 2007). However, these results were based on the comparison between patients with psychosis and healthy controls, and therefore could not establish the diagnostic specificity of the observed changes. Here we extend the previous knowledge by showing that aberrant functional connectivity between the anterior cingulate cortex and the CEN is a distinctive feature of psychosis rather than a transdiagnostic feature of psychiatric illness.

If heightened internetwork correlation between the DMN and the CEN is a distinctive feature of psychosis, one would expect this neural deficit to be associated with a diseasespecific phenotype. Previous studies have suggested that heightened DMN-CEN integration underlies impaired coordination of self-monitoring and task-performance-a core feature of psychosis (Manoliu et al, 2014; Williamson, 2007). Furthermore, existing evidence suggests that DMN-CEN integration is dependent on aberrant input from the insula-a neural mechanism that has a key role in assigning emotional salience to objects, people, and events (Manoliu et al, 2014; Sridharan et al, 2008). This has led to the hypothesis that impaired coordination of self-monitoring and task performance in psychosis can be explained by aberrant emotional salience processing (Manoliu et al, 2014). Further research is required to examine this hypothesis and clarify the phenotypic correlates of the observed heightened correlation between the DMN and the CEN.

A strength of the present investigation is that all four groups were scanned using the same MRI scanner and image acquisition parameters, and therefore the changes in functional connectivity reported here cannot be explained by systematic differences in the acquisition of the data. A further strength is that, at the time of scanning, patients with psychosis were experiencing their first episode of the illness and were still medication-naïve. This means that the alterations in intra- and internetwork functional integration reported here cannot be explained by illness chronicity or medication-two common confounds in neuroimaging studies of psychiatric disease. This may account for the fact that we found fewer and smaller alterations in comparison with previous neuroimaging studies using the same restingstate paradigm (van den Heuvel and Fornito, 2014). For example, as reported in Table 2, the reduced within-network connectivity in the left posterior inferior parietal cortex comprised of a single voxel surviving FWE correction for multiple comparisons. The observation of fewer and smaller alterations in the present investigation suggests that some of the changes reported in the existing literature may reflect the effects of illness chronicity or medication.

In addition, the present investigation has several limitations that are worth mentioning. First, we cannot exclude the possibility that the aberrant functional integration between the DMN and the CEN that we report as a distinctive feature of psychosis may be expressed in other psychiatric groups 
(eg autism). Second, although medication, age, and gender were modelled as covariates of no interest using a linear model, we cannot exclude nonlinear effects of these variables, and furthermore there are other sources of variability such as IQ and wealth status that were not monitored and might have contributed to the results. Third, because we used a cross-sectional rather than a longitudinal design, we were unable to distinguish between correlates of illness vulnerability and correlates of illness onset (Anticevic et al, 2015). Fourth, we used a candidate circuit analysis motivated by the focus of our investigation on three functional networks of interest implicated in psychosis (Menon, 2011); however, the use of alternative analytical approaches such as connectome-wide analysis and topological analysis might have revealed addiction network-level alterations (Fornito and Bullmore, 2015).

In conclusion, our findings indicate a dissociation between aberrant intranetwork connectivity as a transdiagnostic feature of psychiatric illness, and aberrant internetwork connectivity as a distinctive feature of psychosis. They also suggest that, when the confounds of illness chronicity and medication are removed, network-level alterations are less evident than in previous studies. Future research will be required to establish whether the dissociation between intraand internetwork changes reported here is also evident when perform active tasks.

\section{FUNDING AND DISCLOSURE}

The authors declare no conflict of interest.

\section{ACKNOWLEDGMENTS}

This study was supported by the National Natural Science Foundation (Grant Nos. 81220108013, 81371536, 81227002 and 81030027) and Program for Changjiang Scholars and Innovative Research Team (PCSIRT; Grant No. IRT1272). Dr Gong would also like to acknowledge support from his Changjiang Scholar Professorship Award from the Chinese Ministry of Education (Award No. T2014190) and his American CMB Distinguished Professorship Award from the Institute of International Education, USA (Award No. F510000/ G16916411).

\section{REFERENCES}

Adams R, David AS (2007). Patterns of anterior cingulate activation in schizophrenia: a selective review. Neuropsychiatr Dis Treat 3: $87-101$.

Anticevic A, Haut K, Murray JD, Repovs G, Yang GJ, Diehl C et al (2015). Association of thalamic dysconnectivity and conversion to psychosis in youth and young adults at elevated clinical risk. JAMA Psychiatry 72: 882-891.

Ashburner J (2007). A fast diffeomorphic image registration algorithm. NeuroImage 38: 95-113.

Berman RA, Gotts SJ, McAdams HM, Greenstein D, Lalonde F, Clasen L et al (2016). Disrupted sensorimotor and socialcognitive networks underlie symptoms in childhood-onset schizophrenia. Brain 139(Part 1): 276-291.

Braus DF, Ende G, Weber-Fahr W, Demirakca T, Tost H, Henn FA (2002). Functioning and neuronal viability of the anterior cingulate neurons following antipsychotic treatment:
MR-spectroscopic imaging in chronic schizophrenia. Eur Neuropsychopharmacol 12: 145-152.

Du Y, Pearlson GD, Liu J, Sui J, Yu Q, He H et al (2015). A group ICA based framework for evaluating resting fMRI markers when disease categories are unclear: application to schizophrenia, bipolar, and schizoaffective disorders. NeuroImage 122: 272-280.

First M, Spitzer R, Gibbon M, Williams J (1997). Structured Clinical Interview for DSM-IV Axis I Disorders (SCID). American Psychiatric Press: Washington DC.

Fornito A, Bullmore ET (2015). Connectomics: a new paradigm for understanding brain disease. Eur Neuropsychopharmacol 25: 733-748.

Fornito A, Yucel M, Dean B, Wood SJ, Pantelis C (2009). Anatomical abnormalities of the anterior cingulate cortex in schizophrenia: bridging the gap between neuroimaging and neuropathology. Schizophr Bull 35: 973-993.

Friston KJ (1998). The disconnection hypothesis. Schizophr Res 30: 115-125.

Gong Q, He Y (2015). Depression, neuroimaging and connectomics: a selective overview. Biol Psychiatry 77: 223-235.

Jin C, Qi R, Yin Y, Hu X, Duan L, Xu Q et al (2014). Abnormalities in whole-brain functional connectivity observed in treatmentnaive post-traumatic stress disorder patients following an earthquake. Psychol Med 44: 1927-1936.

Kapur S, Phillips AG, Insel TR (2012). Why has it taken so long for biological psychiatry to develop clinical tests and what to do about it? Mol Psychiatry 17: 1174-1179.

Kay S, Opler L, Fiszbein A. (1987). The Positive and Negative Syndrome Scale (PANSS) for schizophrenia. Schizophr Bull 13: 261-276.

Kelly AM, Uddin LQ, Biswal BB, Castellanos FX, Milham MP (2008). Competition between functional brain networks mediates behavioral variability. NeuroImage 39: 527-537.

Littow H, Huossa V, Karjalainen S, Jaaskelainen E, Haapea M, Miettunen J et al (2015). Aberrant functional connectivity in the default mode and central executive networks in subjects with schizophrenia-A Whole-Brain Resting-State ICA Study. Front Psychiatry 6: 26.

Lui S, Wu Q, Qiu L, Yang X, Kuang W, Chan RC et al (2011). Resting-state functional connectivity in treatment-resistant depression. Am J Psychiatry 168: 642-648.

Manoliu A, Riedl V, Zherdin A, Muhlau M, Schwerthoffer D, Scherr $M$ et al (2014). Aberrant dependence of default mode/ central executive network interactions on anterior insular salience network activity in schizophrenia. Schizophr Bull 40: 428-437.

Menon V (2011). Large-scale brain networks and psychopathology: a unifying triple network model. Trends Cogn Sci 15: 483-506.

Menon V, Uddin LQ (2010). Saliency, switching, attention and control: a network model of insula function. Brain Struct Funct 214: 655-667.

Nekovarova T, Fajnerova I, Horacek J, Spaniel F (2014). Bridging disparate symptoms of schizophrenia: a triple network dysfunction theory. Front Behav Neurosci 8: 171.

Pelletier-Baldelli A, Bernard JA, Mittal VA (2015). Intrinsic functional connectivity in salience and default mode networks and aberrant social processes in youth at ultra-high risk for psychosis. PLoS One 10: e0134936.

Pettersson-Yeo W, Allen P, Benetti S, McGuire P, Mechelli A (2011). Dysconnectivity in schizophrenia: where are we now? Neurosci BiobehavRev 35: 1110-1124.

Pettersson-Yeo W, Benetti S, Frisciata S, Catani M, Williams SC, Allen $\mathrm{P}$ et al (2015). Does neuroanatomy account for superior temporal dysfunction in early psychosis? A multimodal MRI investigation. J Psychiatry Neurosci 40: 100-107.

Power JD, Barnes KA, Snyder AZ, Schlaggar BL, Petersen SE (2012). Spurious but systematic correlations in functional connectivity 
MRI networks arise from subject motion. Neurolmage 59: 2142-2154.

Ren W, Lui S, Deng W, Li F, Li M, Huang X et al (2013). Anatomical and functional brain abnormalities in drug-naive first-episode schizophrenia. Am J Psychiatry 170: 1308-1316.

Rubinov M, Bullmore E (2013a). Fledgling pathoconnectomics of psychiatric disorders. Trends Cogn Sci 17: 641-647.

Rubinov M, Bullmore ET (2013b). Schizophrenia and abnormal brain network hubs. Dialog Clin Neurosci 15: 339-349.

Sarpal DK, Robinson DG, Lencz T, Argyelan M, Ikuta T, Karlsgodt $\mathrm{K}$ et al (2015). Antipsychotic treatment and functional connectivity of the striatum in first-episode schizophrenia. JAMA Psychiatry 72: 5-13.

Schmidt A, Diwadkar VA, Smieskova R, Harrisberger F, Lang UE, McGuire $\mathrm{P}$ et al (2014). Approaching a network connectivitydriven classification of the psychosis continuum: a selective review and suggestions for future research. Front Hum Neurosci 8: 1047.

Sridharan D, Levitin DJ, Menon V (2008). A critical role for the right fronto-insular cortex in switching between central-executive and default-mode networks. Proc Natl Acad Sci USA 105: $12569-12574$.

van den Heuvel MP, Fornito A (2014). Brain networks in schizophrenia. Neuropsychol Rev 24: 32-48.

Wang C, Ji F, Hong Z, Poh JS, Krishnan R, Lee J et al (2016). Disrupted salience network functional connectivity and whitematter microstructure in persons at risk for psychosis: findings from the LYRIKS study. Psychol Med 46: 2771-2783.
Weathers F, Litz BT, Huska JA, Keane TM (1994). PTSD Checklist-Civilian Version. National Center for PTSD Behavioural Science Division: Boston, MA, USA.

Williams JB (1988). A structured interview guide for the Hamilton Depression Rating Scale. Arch Gen Psychiatry 45: 742-747.

Williamson P (2007). Are anticorrelated networks in the brain relevant to schizophrenia? Schizophr Bull 33: 994-1003.

Woodward ND, Cascio CJ (2015). Resting-state functional connectivity in psychiatric disorders. JAMA Psychiatry 72: 743-744.

Zhang Y, Xie B, Chen H, Li M, Liu F, Chen H (2016). Abnormal functional connectivity density in post-traumatic stress disorder. Brain Topogr 29: 405-411.

(c) (i) (-) $\ominus$ This work is licensed under a Creative Commons Attribution-NonCommercial-NoDerivs $\quad 4.0$ International License. The images or other third party material in this article are included in the article's Creative Commons license, unless indicated otherwise in the credit line; if the material is not included under the Creative Commons license, users will need to obtain permission from the license holder to reproduce the material. To view a copy of this license, visit http://creativecommons.org/licenses/by-nc-nd/4.0/

(C) The Author(s) 2017

Supplementary Information accompanies the paper on the Neuropsychopharmacology website (http://www.nature.com/npp) 